\title{
Preface: Selected Extended Papers from Interactive Theorem Proving 2018
}

\author{
Jeremy Avigad ${ }^{1}$ (D) Assia Mahboubi ${ }^{2,3}$
}

Received: 15 April 2020 / Accepted: 24 April 2020 / Published online: 22 May 2020

(c) Springer Nature B.V. 2020

The Ninth International Conference on Interactive Theorem Proving (ITP) was held on July 9-12, 2018, in Oxford, UK, as part of the Federated Logic Conference. This special issue of the Journal of Automated Reasoning contains expanded versions of six papers from that conference, chosen by the program committee. All the papers chosen were rated highly in the usual conference review process, but that was not the sole criteria for inclusion. We also looked for work that would benefit from a more expanded, mature treatment, and we aimed for a representative sample of topics.

The expanded versions were reviewed according to the usual standards of this journal. Of the six, two belong to the realm of formalized mathematics and verified mathematical computation:

Christian Doczkal and Damien Pous, "Graph Theory in Coq: Minors, Treewidth, and Isomorphisms"

René Thiemann, Ralph Bottesch, Jose Divasón, Max W. Haslbeck, Sebastiaan J. C. Joosten and Akihisa Yamada, "Formalization the LLL Basis Reduction Algorithm and the LLL Factorization Algorithm in Isabelle/HOL"

The first describes a formal library for graph theory and some important results in that field, whereas the second deals with the Lenstra-Lenstra-Lovász lattice basis reduction algorithm, an important algorithm in algebra and number theory. Three of the papers belong to the realm of hardware and software verification:

Matthew L. Daggitt, Ran Zmigrod and Timothy G. Griffin, "A Relaxation of Üresin \& Dubois Asynchronous Fixed-Point Theory in Agda"

Jeremy Avigad avigad@cmu.edu

Assia Mahboubi assia.mahboubi@inria.fr

1 Department of Philosophy, Carnegie Mellon University, Pittsburgh, PA 15213, USA

2 Inria, LS2N, UFR Sciences et Techniques, 2, rue de la Houssinière, BP 92208, 44322 Nantes Cedex 3, France

3 Vrije Universiteit Amsterdam, Amsterdam, The Netherlands 
Manuel Eberl, Max W. Haslbeck, and Tobias Nipkow, "Verified Analysis of Random Binary Tree Structures"

Hira Taqdees Syeda and Gerwin Klein, "Formal Reasoning under Cached Address Translation"

The first deals with the Üresin-Dubois algorithm, which plays an important role in the correctness of various distributed algorithms, the second deals with the analysis of randomized algorithms, and the third addresses the challenge of verifying low-level operating system code in the presence of caching. Finally, one paper deals with self-verification of interactive theorem provers:

Matthieu Sozeau, Abhishek Anand, Simon Boulier, Cyril Cohen, Yannick Forster, Fabian Kunze, Gregory Malecha, Nicolas Tabareau, and Théo Winterhalter, "The MetaCoq Project"

The paper reports on important progress towards developing a metaprogramming language and support for writing verified tactics and metaprograms.

We are grateful to the authors for enabling us to assemble such a fine collection, and to the anonymous referees for their attention to detail and extremely thoughtful and helpful reviews.

Publisher's Note Springer Nature remains neutral with regard to jurisdictional claims in published maps and institutional affiliations. 